\title{
La atención primaria de salud como fundamento de la reforma de salud salvadoreña
}

\author{
Antonio Hernández Reyes ${ }^{1}$
}

Forma de citar Hernández Reyes A. La atención primaria de salud como fundamento de la reforma de salud salvadoreña. Rev Panam Salud Publica. 2018;42:e130. https:/ / doi.org/10.26633/RPSP.2018.130

RESUMEN Desde la Declaración de Alma-Ata, y durante sus 40 años de vigencia, la atención primaria de salud (APS) se ha convertido en uno de los fundamentos de la reforma de salud salvadoreña iniciada en el 2010. Los valores, principios y elementos de la APS han sido una pieza esencial de su diseño e implementación para alcanzar logros importantes en la salud.

La gratuidad y la ampliación de la cobertura, el ordenamiento de los niveles de atención, el impulso a la promoción de la salud y la voluntad politica se identifican como factores que han permitido la mejora de los indicadores sanitarios, pero no de manera aislada, sino integrados a un proceso de transformación junto a otros programas sociales, que han abonado a la reducción de la pobreza y a la disminución de las desigualdades; estos, a su vez, han contribuido a la mejora de los indicadores de salud.

La reforma de salud debe superar las dificultades actuales que enfrenta, como la deslegitimización frente a la opinión pública, la concepción biologicista de la "buena medicina" y superar aspectos negativos de su gestión como el presupuesto de salud insuficiente y desigual, las condiciones laborales precarias para el personal de salud, el trato deshumanizado y la baja calidad de los servicios. El avance de la reforma estará condicionado a su capacidad de reinventarse, superar las contradicciones que ha generado e ir al encuentro de las necesidades de los trabajadores de salud y la ciudadanía.

Palabras clave Reforma de la atención de salud; atención primaria de salud; políticas públicas de salud; El Salvador.

Hace 40 años se instaló uno de los paradigmas más grandes de la salud mundial conocida como atención primaria de salud (APS) durante una conferencia internacional en Alma-Ata (1978). Su importancia deriva en el reconocimiento de la dimensión política, económica y social de la salud, así como la necesidad de hacerle frente a las desigualdades sanitarias

\footnotetext{
Ministerio de Salud de El Salvador, El Salvador. Enviar correspondencia a Antonio Hernández reyes, antoniohernandezreyes@gmail.com
}

plasmadas en meta de "Salud para todos en el año 2000" (1).

A pesar de que la estrategia de la APS y su mensaje principal se distorsionara en la Región de las Américas debido a los cambios de orientación que adoptaron las agencias internacionales de salud y a los procesos de globalización (2), que la redujeron a programas focalizados (3), su revitalización en el marco de su trigésimo aniversario dio paso a su reincorporación en diferentes países. En la actualidad, se afirma que, con una política de salud basada en la APS que incluya la cobertura financiera universal bajo el control del gobierno o regulada, la distribución equitativa de los recursos, servicios integrales y copagos bajos o nulos para los servicios del primer nivel de atención, se producen mejores niveles de salud (4).

\section{Antecedentes de la reforma y la APS en El Salvador}

Durante y después del conflicto armado en El Salvador, se desarrollaron en el 
país experiencias comunitarias e institucionales exitosas basadas en la APS que no lograron convertirse en política pública. Por el contrario, a finales de los noventa se impulsaron propuestas de reformas neoliberales que desencadenaron una movilización de los médicos, sindicatos, gremiales, y población general en huelgas y marchas blancas ${ }^{2}$ que forzaron una negociación con el gobierno para detener la iniciativa de privatización y la constitución, en 1999, del Consejo de Reforma del Sector Salud (CRSS) como una entidad encargada de formular una reforma del sector con la participación de diferentes entidades públicas y privadas. El CRSS señaló que el sistema de salud salvadoreño mantenía un modelo centralista, excluyente y restrictor del ejercicio democrático en la toma de las decisiones (5).

A partir de ese diagnóstico, se elaboró una propuesta de reforma integral de salud en el año 2000 (6); sin embargo, la falta de voluntad política para implementarla llevó a nuevos conflictos sociales, hasta que se nombró la Comisión Nacional de Seguimiento de la Propuesta de Reforma Integral de Salud, que elaboró algunos documentos regulatorios para el Sistema Nacional de Salud (SNS) pero que no llegaron a materializarse en una política nacional de salud.

En el año 2010, el Ministerio de Salud (MINSAL) lanza la reforma de salud como resultado de más de una década de discusiones y debates entre diferentes sectores que pusieron en el centro del quehacer de salud a la persona humana y su derecho a la salud.

El diseño de la reforma se fundamenta en la APS y retoma sus valores: el derecho a la salud, equidad y solidaridad (2) a través de la instauración la gratuidad en la atención (7). Desarrolla los principios de participación social, responsabilidad y rendición de cuentas e intersectorialidad; esta última a través de la Comisión Intersectorial de Salud (CISALUD) (8) ${ }^{3}$. Pone el énfasis en los elementos de la atención integral, la orientación familiar y comunitaria, la promoción de la salud y la prevención en todos los niveles, mediante la

\footnotetext{
2 "Marchas blancas" fue el término con el que se denominó a las movilizaciones sociales convocadas por el gremio médico para pronunciarse en contra de la privatización de la salud.

3 La Comisión Intersectorial de Salud (CISALUD) es un espacio que involucra a tomadores de decisión de más de 40 instituciones públicas, autónomas, privadas y ciudadanas para el abordaje de la determinación social de la salud.
}

ampliación de la base del primer nivel de atención, aunque con la aclaración de que primer nivel de atención no es sinónimo de APS (9).

Según el último informe de labores del MINSAL (10), se destacan los logros de: a) reducir la tasa de mortalidad materna de 66,29 por cada 100000 nacidos vivos (NV) en 2006 (8) a 27,4 por cada 100000 NV en 2016, con El Salvador como uno de los cinco países con la tasa de mortalidad más baja en la Región (10); b) mejorar las coberturas de vacunación infantil de $89,1 \%$ y $84,7 \%$ en 2008 a $93,9 \%$ y $94,6 \%$ en 2016 en pentavalente y triple viral, respectivamente. (11); c) incrementar la cobertura del parto hospitalario de $43,9 \%$ en 2005 (8) a 99,1\% en 2016 (10); d) reducir en 15 puntos porcentuales la desnutrición crónica de niñas y niños menores de cinco años, de $29 \%$ en 1990 a $14 \%$ en 2014 (10); e) convertirse en el segundo país de la Región con la mejor tasa de lactancia materna exclusiva durante los primeros seis meses de vida de $31,4 \%$ en 2011 a $47 \%$ en 2015 (11) y f) mejorar las coberturas en salud en los municipios más pobres a través de la instalación de los equipos comunitarios de salud familiar (ECOSF), de 59\% en 2007 a $78 \%$ en 2011 (12). Estos ECOSF brindarán acciones de promoción de la salud, preventivas, atención médica y rehabilitación (13). No obstante, hubo algunos retrocesos, como es el caso de la tasa de mortalidad infantil que subió de 7,7 por cada 1000 NV en el año 2008 a 9,8 por cada $1 \mathrm{NV}$ en 2016 (11), en parte por la prevalencia del embarazo en adolescente, que representa, para 2016, 30\% del total de embarazos en El Salvador (10).

\section{La APS como pilar de la reforma}

Está demostrado que aquellos países que fortalecen y amplían su primer nivel de atención mediante la implementación de un modelo con base en la APS logran mejores beneficios en salud (14). Los resultados obtenidos por el proceso de reforma de salud salvadoreña fundamentada en la APS se pueden explicar por cinco factores que se describen a continuación:

1) El establecimiento de la gratuidad y la ampliación de la cobertura del primer nivel de atención. Al eliminar los copagos en los servicios de salud, se disminuyen las barreras económicas $(4,15)$. Para el año 2010, el gasto de bolsillo de las familias salvadoreñas representaba $87 \%$ del gasto privado total en salud (34\% del gasto nacional), mientras que, en el año 2015, esa cifra se redujo a $82 \%$ (correspondiente a $28 \%$ del gasto nacional) $(10,11)$. De esta manera, se incrementaron los egresos hospitalarios en 51,27\% para el año 2011, en comparación con el $2008(12,16)$.

Entre el año 2010 y el 2016, se desplegaron 536 equipos comunitarios, cada uno conformado por un médico, enfermera, auxiliar de enfermería, tres promotores de salud y un polivalente, acompañándose de un aumento significativo del presupuesto del MINSAL hacia el primer nivel de atención (PNA) de $35 \%$ a $38 \%$ (11).

Se reorientaron las contrataciones de recursos humanos entre el PNA y los hospitales. Entre 2008 y 2016 se crearon más de 7000 plazas para trabajadores de salud en los distintos niveles de atención, sobre todo en el PNA, a raíz de la expansión de los ECOSF y las unidades comunitarias de salud familiar (UCSF), las cuales se instalaron en más de la mitad de los municipios del país $(10,11,16)$.

2) Se desmontó el feudalismo hospitalario $^{5}$, que se tradujo en una respuesta eficiente y eficaz de los servicios. La reconfiguración del modelo de gestión por redes integrales e integradas de servicios de salud (RIISS) permitió la reorganización de los servicios y la redefinición de las competencias de los hospitales de segundo y tercer nivel. Las RIISS gestionan los cuidados apropiados de los pacientes desde la puerta de entrada en el PNA hacia los hospitales de mayor complejidad e incrementan la efectividad de las estrategias del enfoque territorial y el registro, evaluación y seguimiento individual (13) de toda la población asignada (16).

Se dotaron de especialistas y subespecialistas médicos según el nivel de complejidad de los hospitales; también se contrataron tocoginecólogos, médicos internistas y pediatras en los 39 ECOS

\footnotetext{
Las unidades comunitarias de salud familiar es el término con que se le denominó a las unidades básicas de atención a nivel local, antes denominadas unidades de salud. Prestan servicios de educación, promoción de la salud, prevención, curación y rehabilitación. Según su nivel de resolución pueden ser básicas, intermedias o especializadas.

5 "Feudalismo hospitalario" es un término introducido por el autor que describe la gestión aislada de un hospital dentro de un sistema de salud, en el cual "avasalla" a sus establecimientos de primer nivel potenciando su funcionamiento (o disfunción) como una entidad independiente (feudo)
} 
especializados instalados en el PNA (11) para descentralizar el control y seguimiento de pacientes.

Este diálogo permanente entre los hospitales y el PNA logró que, en 2016, más de $99 \%$ de los partos se atendieran en el segundo nivel y tercer nivel, según la complejidad del caso, a diferencia de lo que ocurría antes de la implementación de las RIISS $(10,16)$.

3) El impulso a la promoción de la salud: la promoción de los estilos saludables, la intersectorialidad y la participación ciudadana son elementos clave que se desarrollan desde la programación anual de actividades hasta la formulación de políticas.

La CISALUD articula la intersectorialidad a nivel nacional entre instituciones públicas, autónomas, privadas y la población para la prevención y control de enfermedades y emergencias (8). A nivel local, se realiza en diferentes espacios ya establecidos, como las comisiones municipales de protección civil.

Por otra parte, el Foro Nacional de Sa$\operatorname{lud}^{6}$ ha logrado desarrollar consensos para la toma de decisiones estratégicas impulsando la equidad, calidad e inclusión y un abordaje intersectorial con base en la determinación social de la salud mediante un amplio proceso de participación ciudadana propositiva y vigilante en el marco del ejercicio del derecho a la salud (7).

4) La voluntad política que permite la continuidad del proceso. La reforma cuenta con la voluntad política necesaria para financiarla; así, el gasto nacional en salud se incrementó $43 \%$ y pasó de 1270 millones de dólares estadounidenses (US\$) en 2007 a US\$ 1 821,1 millones en 2016, mientras que el gasto nacional en relación al producto interno bruto (PIB) pasó de 6,3\% a 6,8\% en el mismo período $(10,17)$.

5) La transformación del sistema de salud en el contexto de la transformación del país. Las reformas progresistas abarcan todo el ámbito social y no solo la de la salud y tienen un impacto extenso sobre el bienestar social con un decremento de la pobreza, el desempleo y la desigualdad

\footnotetext{
6 El Foro Nacional de Salud es uno de los ejes de la reforma de salud salvadoreña. Se define como un espacio de organización de la ciudadanía en torno al derecho a la salud. No depende de forma orgánica ni administrativa del MINSAL ni recibe financiamiento público. Su liderazgo comunitario ejerce procesos de participación en la formulación de políticas de salud y contraloría social en los diferentes niveles de atención en salud.
}

(15). Los resultados obtenidos por el proceso de reforma no pueden ser interpretados como hechos aislados, sino que siguen una lógica de transformación nacional a través de una serie de programas sociales que se vienen implementando y que procuran mecanismos de redistribución de riqueza y generación de cambios estructurales a largo plazo.

Programas como la alimentación escolar y la entrega del vaso de leche, los uniformes y útiles escolares para los niños, niñas, el programa de apoyo temporal al ingreso, la pensión básica universal para adultos mayores y la entrega de paquetes agrícolas, entre otros, junto a la reforma de salud, han logrado reducir la tasa de pobreza desde 2008 a 2013: la tasa de pobreza por ingresos se redujo de $46,4 \%$ a $34,8 \%$ y la de extrema pobreza, de $15,4 \%$ a $9,1 \%$. De igual forma, el coeficiente de Gini ha disminuido desde 0,47 en 2009 a 0,41 en 2013 (18).

\section{Dificultades y retos de la reforma}

A pesar de estos logros, solo 1,3\% de la población los reconoce como un elemento exitoso de la gestión del actual gobierno (19). Se puede afirmar, entonces, que se cuenta con una reforma exitosa pero que no está en "el corazón de la gente". La reforma tuvo escasa difusión, con mala calidad del servicio (maltrato al paciente, desabastecimiento de medicamentos e insumos médicos, entre otros) y ahora enfrenta un rechazo público.

La percepción sobre el desempeño del MINSAL ha pasado de estar entre las tres primeras instituciones mejor evaluadas a ocupar el puesto número 10 con una calificación de 5,59 en una escala de 1 a 10 (19), la peor en los últimos ocho años. Como señalara Laurell (15), la salud, que ha sido una política legitimadora de los gobiernos progresistas, se ha convertido en un elemento de deslegitimación.

Otra dificultad ha sido no posicionar una comprensión integral y social de la salud ante la población, sino que prevalece la idea de la "buena medicina" como hospitales, asistencia sanitaria, medicamentos y tecnología sanitaria, lo que lleva a tratar la reforma de salud como un asunto técnico y de recursos financieros sin analizar la mejor manera de abordar las necesidades sanitarias de la población (20). A esto se suman fallas gerenciales y administrativas, que trazan nuevos retos impostergables para la reforma, tales como: superar el insuficiente y desigual presupuesto de salud que alimenta inequidades en el financiamiento sanitario entre los pobres y los asalariados; mejorar las condiciones laborales para el personal de salud que se ha deteriorado en los últimos años (infraestructura digna, insumos y equipo de trabajo adecuado, atención a la salud mental del personal, incentivos laborales, entre otros); humanizar el trato y mejorar la calidad de los servicios que incluye eliminar la burocracia administrativa y operativa; continuar el fortalecimiento de la rectoría del MINSAL y elevar la reforma de salud a una política de Estado que garantice su sostenibilidad jurídica.

\section{Consideraciones finales}

Los avances de la reforma de salud salvadoreña muestran que los sistemas públicos y universales logran mejores resultados sanitarios a partir del involucramiento de la ciudadanía en la gestión y contraloría social, la participación de otros sectores más allá de la salud y la convicción de mantener una lucha permanente en contra de las desigualdades sociales. Alcanzar la equidad en las políticas y el sistema de salud es un imperativo. El PNA es el nivel más equitativo de los niveles de atención, ya que es menos costoso y acorta las brechas en salud con las poblaciones marginadas y empobrecidas $(14,17)$.

La reforma de salud salvadoreña podrá avanzar solo si sabe responder mejor a las expectativas de una sociedad cambiante, mediante la implementación de servicios de salud apropiados que satisfagan la demanda y las nuevas necesidades ciudadanas, y si logra entusiasmar a los trabajadores de salud para que vuelvan a acompañar el proceso. Para esto, deberá desarrollar nuevos liderazgos y construir un pacto social amplio por la salud retomando la guía de su brújula: la atención primaria de salud.

Conflicto de intereses Ninguno declarado por los autores.

Declaración Las opiniones expresadas en este manuscrito son responsabilidad de los autores y no reflejan necesariamente los criterios ni las políticas de la RPSP/PAJPH y/o de la OPS. 


\section{REFERENCIAS}

1. Organización Mundial de la Salud (OMS). Informe sobre la salud en el mundo 2008: La atención primaria de salud, más necesaria que nunca. Ginebra: OMS; 2008.

2. Organización Panamericana de la Salud (OPS). Renovación de la atención primaria de salud en las Américas: Documento de posición de la Organización Panamericana de la Salud/Organización Mundial de la Salud. Washington D.C.: OPS; 2007.

3. Stolkiner A, Comes Y, Garbus P. Alcances y potencialidades de la Atención Primaria de la Salud en Argentina. Ciência \& Saúde Coletiva. 2011;16(6):2807-81.

4. Starfield B. Primary care: an increasingly important contributor to effectiveness, equity, and efficiency of health services. SESPAS report 2012. Gac Sanit. 2012;26(S): 20-6.

5. Acosta M, Sáenz M, Gutiérrez B, Bermúdez J. Sistema de salud de El Salvador. Salud Publica Mexico. 2011;53(S2):S188-S196.

6. Consejo de Reforma Integral de Salud. Propuesta de Reforma Integral de Salud. San Salvador: Consejo de Reforma Integral de Salud; 2000

7. Rodríguez M. La Reforma de Salud en El Salvador. San Salvador; 2010. Disponible en: https://www.paho.org/els/index. php?option=com_docman\&view $=$ download\&category_slug=reforma-del-sector-salud\&alias $=1047$-reforma-de-salud-1\&Itemid=364 Acceso el 26 de julio de 2018.
8. Ministerio de Salud de El Salvador. Informe de labores 2010-2011. San Salvador: Ministerio de Salud; 2011.

9. Vignolo J, Vacarezza M, Álvarez C, Sosa A. Niveles de atención, de prevención y atención primaria de la salud. Arch Med Interna. 2011; 33(1):11-14.

10. Ministerio de Salud de El Salvador. Informe de labores 2016-2017. San Salvador: Ministerio de Salud; 2017.

11. López V. Fuentes J. Priegue J. Salazar V. Molina O. Estudio de Pertinencia y Calidad del Gasto Público del Sector Salud de El Salvador, 2010-2016. San Salvador: Agencia de los Estados Unidos para el Desarrollo Internacional; 2017.

12. Organización Panamericana de la Salud (OPS). Análisis de los Avances de la Reforma de Salud en El Salvador en el período 2009-2011. San Salvador: OPS; 2012.

13. Ministerio de Salud de El Salvador. Lineamientos operativos para el funcionamiento de los ECOS familiares y especializados. San Salvador: Ministerio de Salud; 2011.

14. Starfield B, Shi L, Macinko J. Contribution of Primary Care to Health Systems and Health. Milbank Q. 2005;83(3):457-502.

15. Laurell A. Las reformas de salud en América Latina: procesos y resultados. Cuad Relac Labor. 2016;34(2):293-314.

16. Organización Panamericana de la Salud (OPS). Evaluación de los Aportes de la Reforma de Salud en El Salvador al
Desarrollo del Sistema de Salud y los Objetivos de la Cobertura Universal en Salud. San Salvador: OPS; 2012. Disponible en: https://www.paho.org/els/index. php?option=com_docman\&view $=$ dow nload\&category_slug=reportes-1\&alia$\mathrm{s}=1309$-informe-foro-internacional\&Itemid=364 Acceso el 25 de julio de 2018.

17. Organización Panamericana de la Salud (OPS). El Salvador. Estudio de caracterización y análisis de exclusión Social en salud. 2002-2010. San Salvador: OPS, 2011.

18. Secretaría Técnica y de Planificación de El Salvador. Plan Quinquenal de Desarrollo 2014-2019. Santa Tecla: Gobierno de El Salvador; 2015.

19. Instituto Universitario de Opinión Pública de El Salvador (IUDOP). Los salvadoreños evalúan el tercer año de Gobierno de Salvador Sánchez Cerén. San Salvador: IUDOP; 2017. Boletín de Prensa: Año XXXI, No. 3.

20. Laurell A. Políticas de salud en pugna: aseguramiento frente a sistemas universales públicos. Rev Latino-Am Enfermagem. 2016;24:e2668.

Manuscrito recibido el 28 de diciembre de 2017. Aceptado para su publicación, tras revisión, el 3 de agosto de 2018.
ABSTRACT

\section{Primary health care as the foundation for Salvadorian health system reform}

Keywords
The Declaration of Alma-Ata on primary health care (PHC), issued 40 years ago, has become one of the foundations of El Salvador's health system reform, launched in 2010. The values, principles, and elements of PHC have been an essential piece in the design and implementation of the reform, aimed at achieving significant advances in health.

Free services and the expansion of coverage, the organization of the levels of care, the encouragement of health promotion, and political will are identified as facilitating factors in the improvement of health indicators, a phenomenon that has not occurred in isolation but, rather, as part of a process of change involving other social programs that has helped to reduce poverty and inequality; this, in turn, has contributed to the improvement of health indicators.

Health system reform must overcome its current challenges, such as its lack of legitimacy in the eyes of the public and the biologistical concept of "good medicine," as well as negative aspects of management, such as an inadequate and unequal health budget, poor working conditions for health personnel, impersonal care, and poor-quality services.

The progress of the reform will be determined by its capacity for reinvention and its ability to overcome the conflicts it has generated and identify the needs of health workers and citizens alike.

Health care reform; primary health care; public health policy; El Salvador. 
RESUMO

\section{Atenção primária à saúde como pilar da reforma da saúde em El Salvador}

Da sua origem na Declaração de Alma-Ata e nos seus 40 anos de existência, a atenção primária à saúde (APS) se consolidou como um dos pilares da reforma da saúde de El Salvador iniciada em 2010. Os valores, os princípios e os elementos da APS são peças fundamentais do planejamento e implementação para o alcance de grandes conquistas em saúde.

Gratuidade, expansão da cobertura, organização dos níveis de atenção, incentivo à promoção da saúde e vontade política são identificados como fatores que vêm possibilitando melhorar os indicadores de saúde. Não se trata de um efeito isolado, mas integrado a um processo de transformação junto com outros programas sociais que têm contribuído para reduzir a pobreza e as desigualdades. Isso, por sua vez, ajuda a melhorar os indicadores de saúde.

A reforma da saúde precisa superar as atuais dificuldades como a deslegitimização perante a opinião pública e a concepção biologicista de "boa medicina" e enfrentar as deficiências administrativas e de gestão como um orçamento de saúde insuficiente e desigual, condições de trabalho precárias para os profissionais da saúde, o trato desumanizado e a baixa qualidade dos serviços.

O avanço da reforma está condicionado à capacidade de reinventar-se, superar as contradições geradas e atender as necessidades dos profissionais da saúde e da sociedade.

Palavras-chave Reforma dos serviços de saúde; atenção primária à saúde; políticas públicas de saúde; El Salvador. 